\title{
On the synthesis of trimethylamine and pyrrol from coal-gas; and on the occlusion of hydrogen by zinc dust
}

\section{Greville Williams F.R.S.}

To cite this article: Greville Williams F.R.S. (1885) On the synthesis of trimethylamine and pyrrol from coal-gas; and on the occlusion of hydrogen by zinc dust, Philosophical Magazine Series 5, 19:118, 232-236, DOI: 10.1080/14786448508627672

To link to this article: http://dx.doi.org/10.1080/14786448508627672

冓 Published online: 29 Apr 2009.

Submit your article to this journal $[\pi$

Џll Article views: 2

Q View related articles $\sqsubset$ 
The selenium-actinometer consists of a cylinder of selenium prepared on Mr. Graham Bell's plan. Thirty-eight disks of copper are isolated from each other by disks of mica of smaller radius; the groove thus formed is filled with selenium by being rubbed with a rod of this substance. The cylinder being suitably heated, this selenium acquires a greyish aspect, and is ready for use. The even numbers of copper are united by conductors, and the odd numbers also by another set of conductors. By this arrangement not merely is the resistance of the selenium lessened, but we may still increase the delicacy of the apparatus by increasing the number of disks and that of the layers of selonium, while diminishing the resistance of the latter.

The selenium cylinder is insulated by glass supports in the interior of a glass cylinder, which is exhausted so as to protect it from the disturbing influence of obscure heat.

The whole is placed on a support high enough to aroid the effects of light reflected from adjacent objects. In placing the cylinder care is taken to do it so that its axis is parallel to the terrestrial axis. In this way the sun's rays strike the selenium almost perpendicularly at any hour of the day and always illuminate the same portion. By a slight motion in the plane of the meridian the cylinder could each day be brought into such a position that the rays are quite perpendicular.

If, now, we pass a constant current through this apparatus and through a galvanometer, the copper will show by its various deflections all the changes in the illumination of the selenium.

In order to compare these variations we must first adopt a scale : if we suppose the selenium in complete darkness, its resistance will be a maximum, and the deflection of the galvanometer the least. The greatest effect would be to annul the resistance of the selenium; by withdrawing the latter from the circuit we should obtain a greater deflection, which we call 100 or the maximum light. Dividing the interval thus obtained into 100 equal parts, we shall have actinometrical degrees which are always comparable.

In practice the battery to ba used would be that of Clamond. During the determination of the 100 point and of zero the external part of the pole would bs kept at zero. By repeating the operation at different externa? temperatures, we should obtain a table for reducing the actinometrical degree obtained at a given temperature to what it should be if the external part of the pile were at zero.Comptes Rendus, Feb. 2, 1885.

ON THE SYNTHESIS OF TRIMETHYLAMINE AND PYRROL FROM COAL-GAS; AND ON THE OCCLUSION OF HYDROGEN BY ZINC DUST. BY GREVILLE WILLIAMS, F.R.S.

In a paper on "The Action of some Heated Substances on the Organic Sulphides in Coal-Gas," * I mention that on passing coal-

* See 'Journal of Gas Lighting;' vol. xli. pp. 913, 960. 
gas that had not been purified by lime over palladized pumice at a temperature considerably below a red heat, ammonia and distinct traces of a volatile alkaloid were formed. The palladium was charged with hydrogen by passing the coal-gas over it at about $212^{\circ}$ Fahr. At this temperature palladized pumice absorbs bydrogen from the coal-gas; and at a higher temperature gives it off in such a condition as to react upon the vapour of cyanides and produce ammonia and a volatile alkaloid. When the palladium ceased to act--tbat is to say, when ammonia and the alkaloid no longer continued to be formed-the tube containing the palladized pumice was transferred to a trough of boiling water, and the current of gas was kept up until the metal had become activethat is, charged with hydrogen. The water-bath was then removed, and the tube was heated by a very small gas-flame, when the formation of ammonia and the alkaloid again commenced. The quantity of the latter formed from 250 grains of palladized pumice containing 20 per cent. of palladium was, however, so small that I could not procure enough for analysis.

Having found that zinc dust heated to a low temperature in a current of coal-gas rendered hydrogen active in a similar way to palladium, I determined to ascertain if, by using a much larger quantity than I had of palladized punice, the synthesis conld be effected on a sufficient scale to enable me to analyze the products. For this purpose I used the following simple apparatus:-A globular flask was fitted with a cork carrying two tubes, one of which passed to within $\frac{1}{2}$ inch of the bottom; and, at its lower extremity, was protected by a cage of fine copper gauze, to prevent it from being stopped up by the zinc dust. The flask was thea filled with the latter to the commencement of the neck. 'The gas passed through the zinc dust with the greatest ease.

Experiment 1.-The flask arranged in the manner described was heated with a small rose-burner; and, when the tenuperature arrived at about $400^{\circ}$ Fahr., sulphuretted hydrogen was evolved, accompanied by the same peculiar odour that was observed when using palladized pumice. When a rod moistened with hydrochloric acid was brought near the exit, it fumed strongly. The odour was like that of fish. On holding a piece of fir-ivood moistened with hydrochloric acid in the vapour, the red reaction characteristic of pyrrol was distinctly obtained. The vapour was now passed into dilute hydrochloric acid during the night, and in the morning a scarlet ring was formed on the sides of the beaker about $\frac{3}{4}$ inch above the solution. This was probably pyrrol red, produced, in the well-known manner, by decomposition of the pyrrol by the acid.

Experiment 2.-After the preliminary experiment just described, 19 feet of the gas were passed as before; but the products were conveyed into a Geissler's potash-tube containing hydrochloric acid diluted with twice its volume of distilled water. The product was evaporated to dryness; the chloride of ammonium and tarry matters removed; and the hydrochlorate of the base, which was very

Phil. Mag. S. 5. Vol. 19. No. 118. March 1885. R 
deliquescent, was converted into a platinum salt. It weighed $2 \cdot 7$ graius. The smallest trace of this platinum salt gave the fingers an intensely fishy odour. From its great intensity $I$ inferred that the alkaloid was not methylamine, as when working with palladized pumice 1, at one time, thought it might be, remembering Dr. Debus's synthesis of that base by the action of platinum black on prussic acid*. The platinum salt produced in this experiment was not analyzed, but reserved for further purification.

Experiment 3.-The current was kept up for twelve hours, during which 21.51 feet of gas passed. At this point the flask cracked. On cooling it was broken up; and it was found that the zinc had partly melted. On treating it with dilute sulphuric acid, sulphuretted hydrogen was evolved. The platinum salt weighed $4 \cdot 7$ grains. It yielded on ignition 38.83 per cent. of platinum. The corresponding salt of methylamine requires 41.61 per cent. ; that of dimethylamine requires $39 \cdot 53$ : and that of trimethylamine $37 \cdot 20$. It is not unlikely, however, that traces of methylamine or dimethylamine, or both, were present. It should bere be noticed that the errors tend in the direction of too much platinum, owing to the difficulty of removing the last traces of ammonium salt when working on such minute quantities. It was found that ammonia and traces of the alkaloid were formed in this experiment when the zinc dust was cold; but this was not always the case. At $202^{\circ}$ Fahr., the fumes with hydrochloric acid were more obvious : at $226^{\circ}$ they were observed the instant the rod dipped in the acid was brought near the exit tube. At $242^{\circ}$ the fumes were very briskly evolved; and no apparent increase was noticed up to $356^{\circ}$.

Experiment 4.-The gas was passed for 17 hours, to hydrogenate the zinc ; and 7 hours with heat. The chloride of ammonium produced weighed 15 grains ; the platinum salt amounted to $7 \cdot 7$ grains. On ignition it gave 38.40 per cent. of platinum, or nearly the same as in Experiment 3 . The gas passed amounted to 100 feet.

Eaperiment 5.-Here 101 feet of gas gave 2.03 grains of platinum salt, containing 37.04 per cent. of platinum. This only differs by 0.16 per cent. from the number required by theory for trimethylamine. The sal ammoniac formed amounted to $6 \cdot 2$ grains.

Experiment 6.-The volume of the gas used was not taken. Two precipitates of platinum salt were obtained. The first was put aside to be worked up afterwards with some others to obtain greater purity. The second weighed $7 \cdot 2$ grains and afforded $38 \cdot 47$ per cent. of platinum. The chloride of ammonium weighed 1.9 grain.

Experiment 7.- This time 42 feet of gas were passed. Two crops of crystals of platinum salt were obtained, weighing together 8.0 grains. The mixture yielded 38.05 per cent. of platinum; and $2 \cdot 1$ grains of chloride of ammonium were obtained.

In some of the foregoing experiments the whole of the platinum salts were not used in the determinations of the metal. The

* Journ. Chem. Soc. 1863, p. 249. See also T. Fairley, "On the Action of Hydrogen on Organic Polycyanides," Journ. Chem. Soc. 1864, p. 362. 
portions left were therefore mixed, distilled with soda, and the distillate received in hydrochloric acid. The solution was evaporated to dryness, traces of chloride of ammonium were removed, and the solution treated with a very small quantity of platinic chloride. The solution was then allowed to repose until next day; and the platinum salt formed was filtered off and rejected. The filtrate was treated with more platinic chloride dissolved in alcohol. A copious precipitate was obtained. On analysis it gave $14 \cdot 16$ per cent. of carbon. $3 \cdot 79$ of hydrogen, and $37 \cdot 81$ of platinum. Trimethylamine requires 13.57 per cent. of carbon, 3.77 of hydrogen, and 37.20 of platinum. The following is a summary of the above results :-

No. of experiment.

\section{Platinum} Salt

Gas used.

\section{Alkaloid \\ obt. cale. as Trime- thylamine. Grains.} per cent.
1.

,...

2. $\ldots$

3. ....

4. ....

5. $\ldots$

6. ....

7. ....

$\overline{-}$
$21 \cdot 51$
$100 \cdot 00$
$101 \cdot 00$
$42 \cdot 00$

$2 \cdot 70$
$4 \cdot 70$
$7 \cdot 70$
$2 \cdot 03$
$7 \cdot 20$
$8 \cdot 00$

0.6

$1 \cdot 0$

$1 \cdot 7$

0.5

$1 \cdot 6$

$1 \cdot 8$
Grains.

Mixture ....

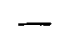

$\begin{array}{rr}- & - \\ - & 38 \cdot 83 \\ \mathbf{1 5 \cdot 0} & 38 \cdot 40 \\ 6 \cdot 2 & 37 \cdot 04 \\ 1 \cdot 9 & 38 \cdot 47 \\ 2 \cdot 1 & 38 \cdot 05 \\ - & 37 \cdot 81\end{array}$

The mean percentage of platinum is 38.10 , which is 0.90 per cent. too high for trimethylamine, and 1.43 per cent. too low for dimethylamine. As the errors of experiment tend to give too high a number for the platinum, I conclude that the base formed was principally trinethylamine. It might also be propylamine, which requires the same numbers, and be formed from cyanide of ethyl as in Mendius's well-known process. Such a supposition, however, does not account for the ammonia formed at the same time$\mathrm{C}_{2} \mathrm{H}_{5} \mathrm{CN}+2 \mathrm{H}_{2}=\mathrm{C}_{3} \mathrm{H}_{8} \mathrm{~N}$. But as the ammonia formed simultaneously with the base is in such large excess, further experiments are necessary to explain all the phenomena. The base butylenediamine, obtained by Fairley from cyanide of ethylene, requires 9.59 per cent. of carbon, $2 \cdot 80$ of hydrogen, and 39.45 per cent. of platinum * ; and moreover would not have the characteristic smell of trimethylamine.

If we assume that the base is formed from prussic acid, the equation becomes $3 \mathrm{CHN}+6 \mathrm{H}_{2}=\mathrm{C}_{3} \mathrm{H}_{9}+2 \mathrm{NH}_{3}$. The production of sal ammoniac in the experiments was too irregular to enable this equation to be confirmed from its amount.

According to Lange $\uparrow$ a polymeride of prussic acid exists having the formula $\mathrm{C}_{3} \mathrm{H}_{3} \mathrm{~N}_{3}$. If formed from this substance, the equation is the same as that given above.

It appeared to me that some light might be thrown upon the

* Fairley, loc. cit. p. $363 . \quad+$ Dent. chem. Ges. Ber. vi. 99. 
question, if hydrogen instead of coal-gas were passed through hydrocyanic acid and then into heated zinc dust; because, if in that case $I$ obtained a number for the platinum in the salt nearer to methylamine, it would show that the formation of trimethylamine from coal-gas did not arise from a peculiarity in the action of the zinc, but from the substance in the gas which gave the alkaloid not being ordinary hydrocyanic acid.

Experiment 8.-Hydrogen prepared from zinc and dilute sulphuric acid was passed through a warm acidulated solution of eyanide of potassium for one day, after a previous passage during the night to render the zinc active. The resulting alkaloid was converted into platinum salt, and wejghed $3 \cdot 20$ grains. It gave on ignition 42.59 per cent. of platinum. The platinum salt of methylamine requires $41 \cdot 68$. Error +0.91 . The chloride of ammonium amounted to $24 \cdot 1$ grains. It is by no means easy to completely separate so small a quantity of methylamine from so much ammonia ; and the excess of platinum is probably due to this cause. The $24 \cdot 1$ grains of chloride of ammonium coutained $7 \cdot 6$ grains of ammonia ; whereas the 3.20 grains of platinum salt of methylamine only contained 0.41 grain of the alkaloid, or less than half a grain. The equation $\mathrm{CHN}+2 \mathrm{H}_{2}=\mathrm{CH}_{5} \mathrm{~N}$ does not involve the formation of any ammonia. If we suppose the base to be formed from cyanogen, we have of course $\mathrm{C}_{2} \mathrm{~N}_{2}+5 \mathrm{H}_{2}=2 \mathrm{CH}_{5} \mathrm{~N}$, which also dispenses with the formation of ammonia.

The smallness of the quantity of alkaloid formed in the reaction (only amounting to $7 \cdot 2$ grains in the six experiments in which it was estimated) would have prevented me from carrying out this preliminary investigation, if I had not, fortunately, had much previous experience in working on minute portions of these substances. The substance or substances which yield ammonia and pyrrol are not entirely removed by treating the gas with lime; as I found in one experiment that, on passing well-purified gas over heated zinc dust rendered active, turmeric paper was reddened and pyrrol evolved.

The source of the pyrrol I hope to clear up by future experiments; but I may mention that I found, in 1855, that it is formed in numerous cases where nitrogenous animal and vegetable matters are subjected to destructive distillation *.

I must not omit to state that, having on one occasion treated zinc dust with very dilute sulphuric acid, with a view to its purification, the washed and dried product refused to act upon the gas in the manner described in the foregoing experiments. I propose to return to this question.-Journal of Gas Lighting, Jan. 6, 1885.

The Gaslight and Coke Company, Nine Elms, December 1884.

- Trans. Roy. Soc. Edin. xxi. part 2. 